\title{
Pharmacogenetics and cognitive symptoms in schizophrenia patients treated with antipsychotics
}

\author{
Richard Musil*,1 \& Ilja Spellmann**,2 \\ ${ }^{1}$ Ludwig-Maximilians University, Department of Psychiatry \& Psychotherapy, Nußbaumstr. 7, 80336 München, Germany \\ ${ }^{2}$ Klinikum Stuttgart, Department of Special Psychiatry, Social Psychiatry \& Psychotherapy, Prießnitzweg 24, 70374 Stuttgart, \\ Germany \\ *Author for correspondence: richard.musi|@med.uni-muenchen.de \\ **Author for correspondence: i.spellmann@klinikum-stuttgart.de
}

\begin{abstract}
"Pharmacogenetic studies on cognitive effects of currently available antipsychotics, although in relatively early stages, suggest that the treatment of cognitive deficits in schizophrenia can be advanced toward a more precise future decision of antipsychotic treatment for individual needs."
\end{abstract}

First draft submitted: 23 May 2018; Accepted for publication: 20 June 2018; Published online: 20 July 2018

Keywords: antipsychotics $\bullet$ cognitive symptoms $\bullet$ pharmacogenetics $\bullet$ schizophrenia

Neurocognitive impairment is considered as a core symptom in schizophrenia [1,2]. A century ago, Kraepelin and Bleuler were among the first clinicians to include impaired cognitive processes in their descriptions of schizophrenia $[3,4]$. They hold the view that the cognitive symptoms of patients have a relatively steady and persistent character regardless of remitted or acute phases of the disease. Its occurrence can precede the first acute episode of the manifest disease by many years and it is estimated that nearly two-thirds of patients show severe cognitive symptoms [5]. However, almost all schizophrenia patients develop cognitive symptoms to a certain degree during the course of the disease [6]. In comparison with other symptomatic dimensions of schizophrenia like positive and negative symptoms, cognitive dysfunctions represent a relatively stable feature and are crucial for the further outcome of the disease and the prospect of achieving remission or even recovery [2,7]. These results have often been replicated and expanded. As a consequence cognitive disorders moved into the focus of research interest for etiopathogenesis and prognosis of schizophrenia [8].

Neurocognitive disorders in schizophrenia include numerous functional domains. An agreement on the presence of deficits in the following cognitive domains was reached at a consensus conference (NIMH-MATRICS Conference): working memory, attention/vigilance, verbal learning and memory, visual learning and memory, reasoning and problem-solving, speed of information processing as well as social cognition [9]. The cognitive deficits in the designated dimensions are mostly from moderate-to-severe expression [10].

Attempts to delineate the pathophysiology of neurocognitive impairment revealed the dorsolateral prefrontal cortex as playing a central role. In the dorsolateral prefrontal cortex dopaminergic neurotransmission is mainly mediated by dopamine D1 receptors. In schizophrenia low levels of dopamine have been observed in this part of the cortex [11].

Furthermore, due to its longitudinal trait stability, neurocognitive symptoms have been considered to be a putative endophenotype in genetic studies [12,13].

Especially polymorphisms in the glutamatergic as well as in the dopaminergic system were associated with different cognitive functions. In the dopaminergic system findings about the COMT gene are relevant [14]. In the glutamatergic system or in its closely related regulation circuits associations between neurocognitive dysfunctions and the following gene polymorphisms were described: DISC1, DTNBP1, NRG1, GAD1, RGS4, GRM3 and G72 genes [15-19].

In recent years, many efforts have been made to positively influence cognitive symptoms. In addition to cognitive remediation therapies, mainly antipsychotics were used, which are presumed to be effective against 
or even modulate the degree of cognitive symptom dimensions [20]. However, the results show only moderate effect sizes in the treatment of cognitive dysfunctions in comparison with other symptomatic dimensions in schizophrenia [20,21]. A possible explanation is that research has focused mainly on 'me-too' style drugs, developing treatments with properties similar to approved treatments. Hence several new antipsychotics were identified and developed with a wider receptor profile (e.g., serotonin 5-HT2A antagonism), but all remained primarily dopamine D2 receptor antagonists [22]. This 'me-too' approach to treatment development limited research investigating the cognitive deficits experienced by people with schizophrenia [20], despite the fact that neurobiological mechanisms of cognitive dysfunctions are complex and include many neurotransmitter systems such as, for example, dopaminergic, glutamatergic and cholinergic pathways [11,23]. New approaches to the treatment of neurocognitive disorders in schizophrenia, including mechanisms such as the stimulation of metabotropic glutamate [24] or acetylcholine receptors with muscarinic and nicotinic receptor agonists $[23,25,26]$ have been investigated, but the results for demonstrating their efficacy have not been conclusive.

Response to treatment is a complex interactional process involving various genetic and nongenetic environmental factors. Nongenetic factors include numerous physiological parameters such as age and gender, liver and kidney function, but also so-called environmental or lifestyle factors such as diet, exercise, alcohol and nicotine consumption. Genetic factors influence these interactions at various levels, as they are involved in pharmacokinetic parameters such as absorption, metabolism and excretion of the substances, but also have a considerable influence on the pharmacodynamics, in other words the level of action on the target proteins.

Although strict approval and proof of efficacy is required in pivotal trials in the registration of antipsychotics, as is the case for all medications, only about $30-60 \%$ of patients respond to treatment with a particular drug [27] and about $7 \%$ develop serious adverse effects [28]. These interindividual differences in response to antipsychotic treatment and its tolerability pose a tremendous challenge to the clinician. It is often similar to a 'trial and error' principle until the patient receives an effective and tolerable drug. In order to minimize this effort, pharmacogenetics tries to identify mutations or so-called genetic polymorphisms that can predict the treatment response or the occurrence of side effects and thus enable a so-called 'tailor-made' or personalized therapy [29]. Although the original concept concerned only the genes that influence the bioavailability of drugs, in psychopharmacological research those genes are now being investigated, which are related to the etiopathology of schizophrenia or are considered as target proteins of psychotropic drugs. For several years, it has also become possible to carry out genome-wide association studies in addition to classical candidate-gene driven study approaches, which make it possible to study millions of SNPs across the entire genome in a hypothesis-free manner with regard to an association with the efficacy and tolerability of pharmaceuticals [28].

To date, only a few molecular genetic studies have been conducted to investigate the effect of antipsychotic treatment on neurocognitive dysfunctions in schizophrenia patients, although neurocognitive dysfunctions as a putative endophenotype of schizophrenia could be a major target criterion [30]. This applies in particular since the execution and evaluation of neuropsychological test procedures can be a well objectifiable, standardized and reliable alternative to the often more subjective assessment of clinical symptoms.

When 21 different response phenotypes with different clinical symptoms were evaluated as criteria of investigation in 756 schizophrenic patients from the Clinical Antipsychotic Trials of Intervention Effectiveness (CATIE) study, the change in cognitive test results over 8 weeks was one of two phenotype-genotype constellations that withstood the study-wide statistical correction of multiple testing. Regardless of which antipsychotic the study subjects had received, those that possessed at least one copy of the rare allele of the polymorphism rs7778604 in the GRM8 gene improved significantly with respect to a task in verbal learning, whereas the homozygous carriers of the more frequent alleles showed a worsening in this psychological test task over 8 weeks [31]. Although the CATIE study was not designed for pharmacogenetic issues this approach may show that changes in neurocognitive symptoms may be a favorable target for pharmacogenetic issues. In the dopaminergic system, the enzyme COMT was investigated in particular with regard to pharmacogenetic issues. One study showed that the genotype COMT Val158Met was able to predict working memory performance and activation of the dorsolateral prefrontal cortex in schizophrenia patients during 8 weeks of olanzapine treatment [32]. Carriers of the Met 158 allele showed better cognitive function as carriers of the Val158 allele variant. In a study by Woodward et al. Met158 allele carriers performed significantly better in executive and working memory performance tests compared with Val158 allele carriers during a 6-month treatment with clozapine [33]. Blasi et al. found that subjects with the rs1076560 $\mathrm{T}$ and rs6314 $\mathrm{T}$ alleles of the dopamine D2 and serotonin 5-HT2A receptor genes have greater fMRI prefrontal activity during working memory [34]. Similar results were obtained within the attentional domain. Furthermore, the concomitant presence 
of the rs $1076560 \mathrm{~T} / \mathrm{rs} 6314 \mathrm{~T}$ alleles also predicted lower behavioral accuracy during working memory. Moreover, they found that rs1076560 T carrier/rs6314 CC individuals had better responses to antipsychotic treatment in two independent samples of patients with schizophrenia. These results indicated that DRD2 and HTR2A genetic variants together may modulate physiological prefrontal efficiency during working memory and also modulate the response to antipsychotics.

Despite the many associations described between candidate genes of the glutamatergic system and schizophrenia, no known study on associations with a special focus on neurocognitive symptoms has so far been published from a pharmacogenetic point of view. In an analysis of data from the CATIE study, significant associations between SNPs in the GRM7 and GRM8 genes and an improvement in neuropsychological test results were shown [31,35], indicating that these genes may be involved in changing neurocognitive dysfunction in schizophrenia patients during antipsychotic treatment. Koola et al. described associations between single nucleotide polymorphisms in the CHRNA7, NMDA, GRM1, DTNBP1 and KMO genes and prediction of treatment response to a galantamine and memantine combination for cognitive impairments in schizophrenia [36].

Neurocognitive symptoms also served as a target in a genome-wide association study of the antipsychotic response in a subset of 738 schizophrenia patients from the CATIE study [37]. In this study, six SNPs localized within or in close proximity to the genes EHF, SLC26A9, DRD2, GPR137B, CHST8 and IL1A were identified and associated with improvements in various neurocognitive domain areas. A significant result was found for the SNP rs286913 in the $E H F$ gene that was associated with the effect of ziprasidone on vigilance. Furthermore, the SNPs rs11240594 in the SLC26A9 gene and rs11677416 in the IL1A gene were associated with the effect of olanzapine on processing speed and working memory. Although the functional mechanisms of these genes on the pathogenesis of schizophrenia and on the psychopharmacological treatment have not yet been elucidated, the authors concluded that the inclusion of neurocognitive symptoms in pharmacogenetic issues may be useful.

Pharmacogenetic studies on cognitive effects of currently available antipsychotics, although in relatively early stages, suggest that the treatment of cognitive deficits in schizophrenia can be advanced toward a more precise future decision of antipsychotic treatment for individual needs. Furthermore, the future development of the area may be enhanced by the use of pharmacogenomic strategies to identify novel factors that influence the response to treatment. In addition to hypothesized candidate gene approaches, genome-wide studies may help identifying new proteins and signaling pathways involved in antipsychotic treatment.

Financial \& competing interests disclosure

The authors have no relevant affiliations or financial involvement with any organization or entity with a financial interest in or financial conflict with the subject matter or materials discussed in the manuscript. This includes employment, consultancies, honoraria, stock ownership or options, expert testimony, grants or patents received or pending, or royalties.

No writing assistance was utilized in the production of this manuscript.

\section{References}

1. Aquila R, Citrome L. Cognitive impairment in schizophrenia: the great unmet need. CNS Spectr. 20(Suppl. 1), 32-40 (2015).

2. Green MF, Harvey PD. Cognition in schizophrenia: past, present and future. Schizophr. Res. Cogn. 1(1), e1-e9 (2014).

3. Bleuler E. Dementia praecox or the group of schizophrenias, Zinkin J. (Ed.) International University Press, NY, USA (1950).

4. Kraepelin E. Dementia Praecox and Paraphrenia. Livingstone, Edinburgh, UK (1919).

5. Lewis R. Should cognitive deficit be a diagnostic criterion for schizophrenia? J. Psychiatry Neurosci. 29(2), 102-113 (2004).

6. Meltzer HY. Cognitive factors in schizophrenia: causes, impact and treatment. CNS Spectr. 9(10 Suppl. 11), 15-24 (2004).

7. Strassnig MT, Raykov T, O'Gorman C et al. Determinants of different aspects of everyday outcome in schizophrenia: the roles of negative symptoms, cognition and functional capacity. Schizophr. Res. 165(1), 76-82 (2015).

8. Keefe RS, Haig GM, Marder SR et al. Report on ISCTM consensus meeting on clinical assessment of response to treatment of cognitive impairment in schizophrenia. Schizophr. Bull. 42(1), 19-33 (2016).

9. Green MF, Nuechterlein $\mathrm{KH}$, Gold JM et al. Approaching a consensus cognitive battery for clinical trials in schizophrenia: the NIMH-MATRICS conference to select cognitive domains and test criteria. Biol. Psychiatry 56(5), 301-307 (2004).

10. Heaton RK, Gladsjo JA, Palmer BW, Kuck J, Marcotte TD, Jeste DV. Stability and course of neuropsychological deficits in schizophrenia. Arch. Gen. Psychiatry 58(1), 24-32 (2001). 
11. Howes O, McCutcheon R, Stone J. Glutamate and dopamine in schizophrenia: an update for the 21 st century. J. Psychopharmacol. 29(2), 97-115 (2015).

12. Corvin A, Donohoe G, Hargreaves A, Gallagher L, Gill M. The cognitive genetics of neuropsychiatric disorders. Curr. Top. Behav. Neurosci. 12, 579-613 (2012).

13. Schmitt A, Malchow B, Keeser D, Falkai P, Hasan A. Neurobiology of schizophrenia: new findings from the structure to the molecules. Nervenarzt 86(3), 324-326, 328-331 (2015).

14. Egan MF, Goldberg TE, Kolachana BS et al. Effect of COMT Val108/158 Met genotype on frontal lobe function and risk for schizophrenia. Proc. Natl Acad. Sci. USA 98(12), 6917-6922 (2001).

15. Addington AM, Gornick M, Duckworth J et al. GAD1 (2q31.1), which encodes glutamic acid decarboxylase (GAD67), is associated with childhood-onset schizophrenia and cortical gray matter volume loss. Mol. Psychiatry 10(6), 581-588 (2005).

16. Callicott JH, Straub RE, Pezawas L et al. Variation in DISC1 affects hippocampal structure and function and increases risk for schizophrenia. Proc. Natl Acad. Sci. USA 102(24), 8627-8632 (2005).

17. Harrison PJ, Law AJ. Neuregulin 1 and schizophrenia: genetics, gene expression and neurobiology. Biol. Psychiatry 60(2), 132-140 (2006).

18. Harrison PJ, Weinberger DR. Schizophrenia genes, gene expression and neuropathology: on the matter of their convergence. Mol. Psychiatry 10(1), 40-68 (2005).

19. Lewis CM, Levinson DF, Wise LH et al. Genome scan meta-analysis of schizophrenia and bipolar disorder, part II: schizophrenia. Am. J. Hum. Genet. 73(1), 34-48 (2003).

20. Young JW, Geyer MA. Developing treatments for cognitive deficits in schizophrenia: the challenge of translation. J. Psychopharmacol. 29(2), 178-196 (2015).

21. Meltzer HY. New trends in the treatment of schizophrenia. CNS Neurol. Disord. Drug Targets 16(8), 900-906 (2017).

22. Richelson E, Souder T. Binding of antipsychotic drugs to human brain receptors: focus on newer generation compounds. Life Sciences 68(1), 29-39 (2000).

23. Mackowick KM, Barr MS, Wing VC, Rabin RA, Ouellet-Plamondon C, George TP. Neurocognitive endophenotypes in schizophrenia: modulation by nicotinic receptor systems. Prog. Neuropsychopharmacol. Biol. Psychiatry 52, 79-85 (2014).

24. Kinon BJ, Zhang L, Millen BA et al. A multicenter, inpatient, Phase II, double-blind, placebo-controlled dose-ranging study of LY2140023 monohydrate in patients with DSM-IV schizophrenia. J. Clin. Psychopharmacol. 31(3), 349-355 (2011).

25. Bradley SR, Lameh J, Ohrmund L et al. AC-260584, an orally bioavailable M(1) muscarinic receptor allosteric agonist, improves cognitive performance in an animal model. Neuropharmacology 58(2), 365-373 (2010).

26. Walling D, Marder SR, Kane J et al. Phase II trial of an $\alpha-7$ nicotinic receptor agonist (TC-5619) in negative and cognitive symptoms of schizophrenia. Schizophr. Bull. 42(2), 335-343 (2016).

27. Spear BB, Heath-Chiozzi M, Huff J. Clinical application of pharmacogenetics. Trends Mol. Med. 7(5), 201-204 (2001).

28. Pouget JG, Shams TA, Tiwari AK, Muller DJ. Pharmacogenetics and outcome with antipsychotic drugs. Dialogues Clin. Neurosci. 16(4), 555-566 (2014).

29. Hamburg MA, Collins FS. The path to personalized medicine. N. Engl. J. Med. 363(4), 301-304 (2010).

30. Burdick KE, Gopin CB, Malhotra AK. Pharmacogenetic approaches to cognitive enhancement in schizophrenia. Harv. Rev. Psychiatry 19(3), 102-108 (2011).

31. Need AC, Attix DK, McEvoy JM et al. A genome-wide study of common SNPs and CNVs in cognitive performance in the CANTAB. Hum. Mol. Genet. 18(23), 4650-4661 (2009).

32. Bertolino A, Caforio G, Blasi G et al. Interaction of COMT (Val(108/158)Met) genotype and olanzapine treatment on prefrontal cortical function in patients with schizophrenia. Am. J. Psychiatry 161(10), 1798-1805 (2004).

33. Woodward ND, Jayathilake K, Meltzer HY. COMT val108/158met genotype, cognitive function and cognitive improvement with clozapine in schizophrenia. Schizophr. Res. 90(1-3), 86-96 (2007).

34. Blasi G, Selvaggi P, Fazio L et al. Variation in dopamine D2 and serotonin 5-HT2A receptor genes is associated with working memory processing and response to treatment with antipsychotics. Neuropsychopharmacology 40(7), 1600-1608 (2015).

35. Need AC, Keefe RS, Ge D et al. Pharmacogenetics of antipsychotic response in the CATIE trial: a candidate gene analysis. Eur. J. Hum. Genet. 17(7), 946-957 (2009).

36. Koola MM. Kynurenine pathway and cognitive impairments in schizophrenia: pharmacogenetics of galantamine and memantine. Schizophr. Res. Cogn. 4, 4-9 (2016).

37. McClay JL, Adkins DE, Aberg K et al. Genome-wide pharmacogenomic study of neurocognition as an indicator of antipsychotic treatment response in schizophrenia. Neuropsychopharmacology 36(3), 616-626 (2011). 